\title{
SCAFFOLD-IN-SCAFFOLD POTENTIAL TO INDUCE GROWTH AND DIFFERENTIATION OF CARDIAC PROGENITOR CELLS
}

\author{
MATTEO $\mathrm{CIOCCI}^{1^{*}}$, FEDERICO MOCHI ${ }^{2 *}$, FELICIA CAROTENUTO ${ }^{3,4^{*}}$, EMILIA DI GIOVANNI ${ }^{1}$, \\ PAOLO PROSPOSITO ${ }^{2,3}$, ROBERTO FRANCINI ${ }^{2,3}$, FABIO DE MATTEIS ${ }^{2,3}$, IGOR RESHETOV ${ }^{3,5}$, \\ MAURO CASALBONI ${ }^{2,3^{*}}$, SONIA MELINO ${ }^{1,3^{*}}$, PAOLO DI NARDO ${ }^{3,4^{*}}$
}

\begin{abstract}
${ }^{1}$ Department of Chemical Sciences and Technology, University of Rome Tor Vergata, via della Ricerca Scientifica, 1, 00133; ${ }^{2}$ Department of Industrial Engineering, University of Rome Tor Vergata, Via del Politecnico 1, 00133 Rome, Italy; ${ }^{3}$ Center for Regenerative Medicine, University of Rome Tor Vergata; Rome, Italy; ${ }^{4}$ Department of Clinical Science and Translational Medicine, University of Rome Tor Vergata, Via Montpellier 1, 00133 Rome, Italy; ${ }^{5}$ Plastic Surgery Chair, Sechenov First Moscow State Medical University, Moscow, Russia.
\end{abstract}

*These authors have equally contributed to the study in their respective roles

Address correspondence to:

Paolo Di Nardo, MD

Center for Regenerative Medicine

University of Rome Tor Vergata

Via Montpellier, 1

00133 Roma, Italy

Phone: +3906 72594215

E-mail:dinardo@uniroma2.it 


\section{Abstract}

The design of reliable biocompatible and biodegradable scaffolds remains one of the most important challenges for tissue engineering. In fact, properly designed scaffolds must display an adequate and interconnected porosity to facilitate cell spreading and colonization of the inner layers, and must release physical signals concurring to modulate cell function to ultimately drive cell fate. In the present study, a combination of optimal mechanical and biochemical properties has been considered to design a one-component 3D multitextured hydrogel scaffold in order to favor cell-scaffold interactions. A PEGDa woodpile (PEGDa-Wp) structure of the order of hundred micrometers has been manufactured using a micro-stereolithography process. Subsequently, the PEGDa-Wp has been embedded in a PEGDa hydrogel to obtain a 3D scaffold-in-scaffold system (3D-SS). Finally, the 3D-SS capability to address cell fate has been assessed using human $\operatorname{Lin}^{-}$Sca- $1^{+}$ cardiac progenitor cells ( $\mathrm{hCPC}$ ). Results have shown that a multitextured 3D scaffold represents a favorable microenvironment to promote $\mathrm{hCPC}$ differentiation and orientation. In fact, while cultured on 3D-SS, hCPC adopt an ordered 3D spatial orientation and activate the expression of structural proteins, such as the ?-sarcomeric actinin, a specific marker of the cardiomyocyte phenotype, and connexin 43, the principal gap junction protein of the heart. Although preliminary, the present study demonstrates that complex multitextured scaffolds closely mimicking the extracellular matrix structure and function are efficient in driving progenitor cell fate. A leap forward will be determined by the use of advanced 3D printing technologies that will improve multitextured scaffold manufacturing and their biological efficiency.

Keywords: cardiac progenitor cells, differentiation, 3D-printing, microstereolithography, biocompatible scaffold, PEGDa hydrogel. 
Introduction

Despite relevant investments and undoubted achievements, cell therapy and tissue engineering represent a not yet accomplished endeavour [i, ii $]$. Novel strategies and materials are needed to fulfill the multifaceted complexity of engineered tissue manufacturing, in which the optimal combination of cells, biocompatible scaffolds and biologically active molecules are required [iii]. In this context, a particular attention must be paid to the design of efficient polymeric scaffolds [iv ${ }^{i}$ on which cells could be grown and differentiated and the tissue bio-architecture addressed. Scaffolds must be immunologically inert, easy to handle, biocompatible and biodegradable. At the same time, they should favor the attachment, growth and migration of cells, and address in situ vascularization by tuning its biochemical and physical characteristics [ $\left.{ }^{\mathrm{V}}\right]$. Finally, an ideal scaffold should display an adequate and interconnected porosity to facilitate cell spreading and colonization of the inner layers. A proper interaction between scaffolds and cells contributes to modulate gene expression and protein organization in cell fate determination [ ${ }^{\text {vi }}{ }^{\mathrm{vii}}$ ]. Engler et al. [ ${ }^{\text {viii] }}$ showed that skeletal myoblasts striated only when cultured on gels with a stiffness mimicking that of the native tissue; cardiac progenitor cells also modulate their cardiac commitment depending on the substrate stiffness and pore geometry $\left[{ }^{i x},{ }^{x}\right]$. Recently, poly(glycerolsebacate) (PGS) scaffolds, with accordion-like honeycomb pores obtained by laser-microablated PGS membranes, were stacked and bonded to produce a 3D mechanically stable pore architecture to guide the alignment of neonatal rat heart cells $\left[{ }^{\mathrm{xi}}\right]$.

In search of the optimal scaffold, innumerable materials and procedures have been proposed for their fabrication. Natural, synthetic and composite materials have been analyzed for tissue repair and regeneration and different strategies to functionalize them have been scrutinized [xii]. Natural materials, although apparently ideal, are difficult to be extracted and manipulated. They are expensive and potentially carriers of infectious agents. Synthetic materials are easily produced at a low cost, but can release by-products that can modify the microenvironment of the recipient tissue. All biomaterials so far analyzed have been tested using different fabrication procedures like electrospinning, freeze-drying, in situ forming gel, direct writing and printing of photopolymerizable 
materials [xiii]. Among others, microstereolithography $(\mu \mathrm{SL})\left[^{\mathrm{xiv}},{ }^{\mathrm{xv}}\right]$, a simple technique for 3D modelling and printing, holds promise to allow to easily manufacture suitable scaffolds for tissue engineering. $\mu S L$ is based on the photo-polymerization of a cross-linkable liquid resin by means of a dynamic photo-mask approach. In this way, it is possible to provide a reconfigurable illumination on the separation surface of a photopolymerizable fluid containing a liquid filter to limit the penetration depth of the light and to ensure a real 3D fabrication. $\mu \mathrm{SL}$ offers far shorter manufacturing times, low cost equipment and materials, and the possibility to easily modify the output structures [ $\left.{ }^{\mathrm{xvi}}\right]$. The above mentioned approaches represent a preliminary stage towards the fabrication of biomimetic structures resembling living tissues in their composite bio-architecture characterized by interlaced cells and materials to maximize the performance of the final assembly. In this context, the intercellular space is filled up with an interstitial matrix in which are embedded fibrillar proteins (mostly collagen) to supply resident cells with structural support. Depending on collagen and elastin concentrations, the ECM can be considered as a collation of different microenvironments displaying variable degrees of stiffness and other physical characteristics influencing numerous cell functions. Therefore, biological tissues could be better mimicked using multitextured materials that incorporate a single or multiple components in order to deliver an appropriate array of physical signals to the cells.

In the present study, a biocompatible polyethylene glycol diacrylate woodpile structure (PEGDa-Wp) was manufactured by [SL using Curcumin as absorbing dye. Subsequently, PEGDa-Wp structures were embedded in a PEGDa hydrogel to better mimic the extracellular matrix microarchitecture creating an inhomogeneous multitextured structure with improved mechano-physical and biological properties. The $\mathrm{Wp}$ architecture represents a reliable structure that allows large deformations with a good specific energy dissipation capacity. Therefore, the optimal combination of this type of in vitro 3D scaffolds and PEGDa-hydrogel was expected to favor cell-cell and cell-material interactions, and to promote the differentiation of the human $\mathrm{Lin}^{-} \mathrm{Sca}^{+}$cardiac progenitor stem cells (hCPCs). This study allowed to validate the novel concept of multitextured 3D"scaffold in scaffold" (3D-SS), in which 3D structures manufactured on the basis of diverse designs could be combined to obtain scaffolds with unprecedented dynamic 


\section{Page 5 of 32

อ

电

characteristics. This study represents the first demonstration of the feasibility of this strategy that can be further enhanced by the application of the emerging 3D-bioprinting 苍 technologies. 


\section{PEGDa-Woodpile fabrication by ?SL}

The precursor solution used to manufacture the $\mathrm{Wp}$ structure was composed by a hydrogel of PEGDa MW $575 \mathrm{~g} / \mathrm{mol}$, Curcumin as absorbing dye (inner optical filter) and 2Hydroxy-4-(2- hydroxyethoxy)-2-methylpropiophenone (also known as Irgacure $819{ }^{\circledR} \mathrm{CIBA}$ ) as photoinitiator. All reagents were purchased from Sigma Aldrich and used without further purification. Curcumin and Irgacure at a concentration of $44 \mathrm{mM}$ and $0.25 \mathrm{mM}$, respectively, were dissolved in a PEGDa:ethanol = 3:1 solution. After mixing, the solutions were let stir for $24 \mathrm{~h}$ in the darkness. The 2D models (images) to be printed layer by layer, drown with a standard software, were projected and focused by means of an overhead projector on the printing stage covered by a thin film of precursor solution. Polymerization occurred only in the lit area during the exposition. After such an exposition, the printed structure was formed on the stage. Then the platform was lowered down in the liquid solution (by a fixed step of $250 \mu \mathrm{m}$ ) and a new thin layer of unpolymerized solution covered it, ready to be exposed. The inner optical filter (curcumin) ensured that only the top layer received enough light to photopolymerize, while the previously exposed layers remained protected against further photo-polymerization due to the absorption of curcumin. The orientation of the polymerized rods was alternated in the subsequent layers and each couple of orthogonally oriented layers could be considered as a monolayer of the structure. The final 3D-template resulted as the stacking of the sequence of all printed 2D layers. A schematic representation of the experimental 3D printing set-up is shown in Fig. (Fig. 1). At the end of the process, the scaffolds were accurately washed in water and cured under Hg UV-lamp for few minutes to stabilize the structure against uncontrolled modification induced by room light. This procedure allowed obtaining structures with a resolution of about $300 \mu \mathrm{m}$; they were morphologically characterized by an optical microscope (Nikon L-IM equipped with a digital camera DS-5M), while their stiffness was assessed by a nanoindenter (Nano Test Micro Materials Ltd.), using a diamond Berkovitch tip. 
Before further steps, the PEGDa-Wp structures were washed with sterile water for one week and exposed overnight to the UV light. They were then conditioned for one week in Dulbecco's Modified Eagle Medium (DMEM) with high glucose (Gibco, Italy) supplemented with $20 \%$ v/v Fetal Bovine Serum (FBS) (Gibco, Italy), 1\% penicillin-streptomycin (SigmaAldrich, Italy), 1\% L-Glutamine (Gibco, Italy) and 1\% non-essential amino acids solution (Sigma-Aldrich, Italy). Finally, the scaffolds were used for cell cultures.

\section{D-SS fabrication}

The 3D-SS systems were manufactured by embedding the PEGDa-Wp into a PEGDa hydrogel obtained by photopolymerization of a solution of 15\% w/v PEGDa $20 \mathrm{kDa}$ MW and $3 \% \mathrm{w} / \mathrm{v}$ PEGDa $6 \mathrm{kDa} \mathrm{MW}$ in phosphate buffered saline (PBS). The hydrogel photopolymerization was achieved using molds with $5 \mathrm{~mm}$ diameter and adding $0.1 \% \mathrm{w} / \mathrm{v}$ Irgacure 2959 (Ciba Specialty Chemicals, Basel, Switzerland). Finally, the samples were exposed to UV light $\left(365 \mathrm{~nm}, 4-5 \mathrm{~mW} \mathrm{~cm}^{-2}\right)$ for $5 \mathrm{~min}$.

\section{Cell culture and Cell proliferation assay}

Human Lin-Sca-1+ cardiac progenitor cells (hCPCs) were isolated from samples of the right auricles of patients (seven male, six female, 52-83 years) undergoing coronary artery bypass surgery [xvii] after signing a written consent form according to a joint protocol approved by the Ethic Committee of Ospedale Maggiore della Carita', Novara, Italy. hCPCs ( $1 \times 10^{4}$ cells in $50 \mu$ l of DMEM) were seeded and cultured on PEGDa-Wp in DMEM with high glucose containing 10\% FBS, 1\% penicillin-streptomycin, 1\% L-Glutamine. Cell cultures in 3D-SS systems were performed seeding $4.2 \times 10^{5} \mathrm{cell} / \mathrm{ml}$ of hCPCs in a total volume of $60 \mu \mathrm{l}$ of PEGDa hydrogel precursor mixture. After cell seeding, the mixture was polymerized under UV light, as described above. The cells into the 3D-SS systems were cultured in a complete medium for 3 weeks. The proliferation and viability of hCPCs were analyzed using a Water-Soluble Tetrazolium salt (WST-1) assay (4-[3-(4-lodophenyl)-2-(4nitrophenyl)-2H-5-tetrazolio]-1,3-benzene disulfonate) (Roche Diagnostics, Sigma Aldrich, 
Italy). Briefly, the samples were incubated for $3 \mathrm{~h}$ in DMEM high glucose without phenolred supplemented with $10 \%$ FBS, $1 \%$ penicillin-streptomycin, $1 \%$ L-Glutamine and in the presence of 5\% v/v WST-.1. Subsequently, the absorbance of the medium was evaluated using iMark ${ }^{\mathrm{TM}}$ Microplate Reader (Bio-Rad) at a wavelength of $450 \mathrm{~nm}$. The number of the hCPCs attached to the PEGDa-Wp was evaluated by the absorbance at $450 \mathrm{~nm}$ after WSTassay, using a calibration curve obtained culturing different numbers of cells from 0 to $10^{5}$ cells (Fig. 1S).

\section{Recognition of the hCPC phenotype when cultured on 3D-SS}

To assess the hCPC phenotype, 3D-SS with $1 \times 10^{4}$ cells Lin ${ }^{-S c a-1}{ }^{\text {pos }}$ hCPCs were cultured for $7 \mathrm{~d}$ in complete medium. The scaffolds were then washed in PBS, fixed in 4\% PFA in PBS for $15 \mathrm{~min}$ at $4^{\circ} \mathrm{C}$, permeabilized with $0.2 \% \mathrm{v} / \mathrm{v}$ Triton X-100 (Sigma-Aldrich, Italy) for 10 min and incubated with phalloidin-Alexa fluorochrome-conjugated (Life Technologies, Italy). In a further set of experiments, the hCPC phenotype on 3D-SS systems was assessed after incubation with cardiac alpha sarcomeric actinin antibody (Ab-??-actinin, SigmaAldrich, Italy) for $9 \mathrm{~h}$ and with the secondary antibody (Goat anti-Mouse IgG $(\mathrm{H}+\mathrm{L})$, Alexa Fluor $^{\circledR} 546$, ThermoFisher) for $4 \mathrm{~h}$ at room temperature. Nuclei were stained with Hoechst 33342 (Sigma-Aldrich, Italy). Confocal microscopy of the cell-seeded constructs was performed using a LSM 700 Confocal Laser Scanning Microscope (Carl Zeiss Microlmaging, Jena, Germany) and acquired by means of ZEN 2010 software.

The differentiating potential of the PEGDA-Wp structure (in the absence of hydrogel) was assessed by seeding hCPCs onto sterile 2D PEGDa-Wp and 3D PEGDa-Wp structures in DMEM supplemented with $10 \%$ FBS. One days after seeding the cells were shifted to DMEM supplemented with $2 \%$ FBS. hCPCs were kept in culture for further eleven or twenty days. The medium was changed every 2 days. hCPCs cultured in the same condition in the chamber slides in the absence of structures represented the control. The hCPCs cultured in the absence or presence of PEGDa structures were fixed with cold methanol and the expression of the cardiac alpha sarcomeric actinin ( $\alpha$-actinin) and connexin-43 (Cx43) were assessed after incubation with specific primary antibodies (Sigma-Aldrich) for 


\section{Page 9 of 32}

$1 \mathrm{~h}$ at RT and then with the appropriate anti-mouse or anti-rabbit secondary antibodies (Alexa Fluor ${ }^{\circledR} 546$, ThermoFisher) for $1 \mathrm{~h}$ at RT. The secondary antibody in the absence of specific primary antibody was used to exclude the occurrence of unspecific signals. Nuclei were stained with 1 l $\mathrm{g} / \mathrm{ml}$ 4',6-diamidino-2-phenylindole (DAPI) (Sigma-Aldrich). The confocal images were taken using a confocal laser scanning microscopy Olympus Fluoview 1000.

\section{Statistical analysis}

The statistical analysis was performed using GraphPad Prism version 5.0 for Windows (GraphPad Software, San Diego, CA, USA). Data from three or five independent experiments were quantified and analyzed for each variable using a one-tailed Student's ttest or ANOVA One-way test. A P-value of $<0.05$ was considered to be statistically significant. Standard deviations of the mean were calculated and presented for each type of sample. 


\section{Results}

\section{Scaffold fabrication and characterization}

In microstereolithography, the most crucial aspect is the choice of the inner optical filter. It must almost totally absorb the incoming light in a very thin layer. Indeed, growing an object layer-by-layer is possible only if the exposition of each new layer does not affect the already exposed lower structure. A careful choice of dye and its concentration are mandatory in order to reduce the light to a negligible amount after 250 ?m corresponding to the desired growth step. To this end and considering the biological context of the application, the photopolymerization has been carried out using a precursor solution with curcumin, a natural dye extracted from Curcuma longa spice widely used in Middle- and Far-East Asian areas, displaying a total absorption coefficient of $100 \mathrm{~cm}^{-1}$ in the active spectral region (350-470nm). Curcumin absorption matches well the absorption spectrum of the Irgacure 819, the photoinitiator used in the present study, in the spectral interval of the lamp (see Fig. 2S) while its luminescence does not overlap Irgacure absorption. Moreover, it is endowed with well-known anti-oxidants, anti-inflammatory and antitumoral properties [ $\left.{ }^{\text {xiii }}\right]$. The dye and the photoinitiator were dissolved in the precursor solution with a ratio of about 200:1 in order to confine the photopolymerization in a thin layer only, just below the exposed surface.

All manufactured PEGDa scaffolds were preliminary characterized by optical microscopy. Fig. $1 \mathrm{C}$ shows great regularity in accordance with the planned structure sketched in the inset. The average thickness of the segments of the PEGDa-Wp scaffold used in the present study is about $300 \mu \mathrm{m}$. The mechanical characterization of the PEGDa was accomplished with a nanoindenter. The ratio of the uniaxial stress to the uniaxial strain of a material, the Young modulus, can be experimentally determined from the slope of linear fits to the stress-strain curves in an indentation experiment $\left[{ }^{\mathrm{xix}}\right]$. Nanoindentation test was performed on a $5 \mathrm{~mm}$ diameter disk of $2.5 \mathrm{~mm}$-thick bulk PEGDa samples grown by the same $\mu S L$ technique repeating the projection of the disc for ten layers. The measurement of a dried sample yielded a value of the elastic modulus of $5 \mathrm{MPa}$. A hydrated sample, whose surface was blown in a gentle stream of air prior to 
nanoindentation experiments to remove the excess water, was measured yielding a value of $1 \mathrm{MPa}$. The results are in full accordance with Drira et al. $\left[{ }^{\mathrm{xx}}\right]$. Noteworthily, depending on the morphology of the tridimensional cage, the mechanical properties of the entire $\mathrm{Wp}$ structures could be greatly different from those related to the bulk material only.

\section{hCPCs growth and differentiation on PEGDa-WP structures}

hCPCs $\left(1 \times 10^{4}\right.$ cells $\left./ \mathrm{cm}^{2}\right)$ were seeded on PEGDa-Wp structures (Fig. $\left.2 \mathrm{~A}\right)$; after $12 \mathrm{~h}$, the structures were transferred in new wells in order to analyze the proliferation of cells actually attached. Cell viability and proliferation were assessed by WST assay after 4 days of cell growing (Fig. 2B): about $4 \times 10^{4}$ viable cells were found attached to each scaffold (Fig. 2C). After eight days of cell growth, the microscope analysis showed that the adhesion to the PEG-Wp was quite good and the cells were mostly clustered around the scaffold struts (Fig. 3). In particular, the confocal microscopy (Fig. 3C, D) showed a spindled and branched morphology of the hCPCs suggesting a potential effect of the scaffold structure on cell assembly and fate.

The PEGDa-Wp differentiating potential was assessed seeding hCPCs onto the structures that, after one day, were shifted in new wells and cultured with DMEM supplemented with $2 \%$ of FBS for eleven days. The evidence of a possible hCPCS differentiation towards a cardiomyocytic phenotype was achieved by assessing the expression of specific cardiac differentiation markers, such as $\alpha$-actinin and Cx43. Fig. 4 shows the confocal micrographs of the ?-actinin expression in hCPCs seeded on a plate in the absence of the structure (Fig. 4A) and in the presence of 2D PEGDa-Wp (Fig. 5B) or 3D PEGDa-Wp (Fig. 4C). Immunostaining revealed that the ?-sarcomeric actinin was widely expressed only in hCPCs cultured in the presence of the microlythographed 3D PEGDa (Fig. 4C) implying that the microstructured scaffold could induce the progenitor cell differentiation towards a cardiomyocytic phenotype. Noteworthily, the microstructure contributed to direct cell spatial-ordered multilayer organization. In addition, Fig. 5 B shows that $\mathrm{C} \times 43$, the principal gap junction protein of the heart, was highly expressed in the cytoplasm of hCPCs cultured on microlythographed 3D PEGDa (Fig.5 C) rather than in cells cultured on 2D structures (Fig. 5A and B). 


\section{hCPCS growth and differentiation into 3D scaffold-in-scaffolds}

In the present study, a novel multitextured hydrogel scaffold 3D-SS was manufactured combining a PEGDa-Wp structure with a PEGDa hydrogel mold. $2.5 \times 10^{4}$ hCPCs were seeded into 3D-SS and, after three weeks of growth with DMEM supplemented with $10 \%$ of FBS, cell viability was checked by WST-1 assay (Fig. 6A), while cell differentiation was analyzed by immunostaining using Ab-anti-? ?-actinin (Fig. 6B). In Figures 6B and C, the confocal observation of the hCPCs growing into 3D-SS demonstrated an upregulation of ?actinin, whose expression was undetectable in 2-D control cultures (Fig. 3S); in addition, cells adhering to the construct displayed an elongated morphology. Therefore, the cell alignment on the PEGDa-Wp (Fig. 6B and D) and the expression of ?-actinin demonstrated the differentiating potential of the PEGDa-Wp in a more complex system. Thus, 3D-SS was able to stimulate correct stem cell commitment towards cardiac lineage, although a complete sarcomeric rearrangement in these cells was not observed. Figure 7 summarizes the entire 3D-SS/ hCPCs procedure. 


\section{Discussion}

The present study demonstrates that biologically efficient multitextured scaffolds mimicking the inhomogeneous microarchitecture of the extracellular matrix can be manufactured by combining structures with different physical characteristics. The proofof-concept has been achieved by embedding a stiffer woodpile structure into a softer hydrogel (scaffold-in-scaffold) in order to provide cells with a resorbable mechanical support and orientation grid for their 3D growth, differentiation and alignment in a microenvironment rich in oxygen, water and metabolites flowing through interconnected channel networks also draining the products of cell catabolism. Indeed, the ECM is made of the matrix in which filaments of different size, structure and physical characteristics are embedded $\left[{ }^{x x i}, x x i\right]$. The validation of this approach has been obtained growing cardiac progenitor cells in the scaffold-in-scaffold system: cells three-dimensionally aligned along the three-axes of the scaffold and triggered their differentiation towards the myocyte phenotype. These results pave the way for manufacturing multicomponent composite structures more efficiently mimicking the three-dimensional organization and function of the extracellular matrix. The goal is to manufacture scaffolds in which multiple specifically tuned "stiffer" structures are combined with several "softer" materials characterized by variable textures (pore number, geometry and interconnection, etc) [ ${ }^{\text {xiii }}$ (Fig. 6). Furthermore, the heterogeneous scaffolds could be enriched with biologically active molecules (cytokines, growth factors, antibodies, etc.) or delivery systems (microbubbles, microbeads, exosomes, etc.) $\left.{ }^{[x i v}\right]$ able to convey signals on the basis of a predetermined spatiotemporal schedule.

Many aspects of engineered tissue manufacturing remain to be clarified yet, before a safe and efficient application to the clinical setting could be envisaged. Several materials and procedures to manufacture the scaffolds, on which cells can be grown and differentiated, have been proposed, but optimal solutions are not yet at hand. In general, two major classes of materials have been so far considered to manufacture scaffolds. In the first class are materials extracted from biological tissues (mostly components of the extracellular matrix) $\left.{ }^{\mathrm{xxv}}\right]$ : they are expensive and not completely safe, since it cannot be prevented that noxious agents could be transferred to the recipient subject. A particular 
subclass of natural materials is represented by perfusion-decellularized human hearts from which it is possible to obtain acellular cardiac scaffolds with preserved ECM properties, structure, patent coronary conduits, with an immunologic profile that induces a constructive remodeling response $\left[{ }^{\mathrm{xxv}}\right]$. However, this technologically superb solution does not phase out the need of a donor and the possibility that some viral copies could be passed via the scaffold [ ${ }^{\text {xxvii] }}$. To avoid these limits, the only possibility is to learn how to manufacture a suitable scaffold using synthetic or semi-synthetic materials. Indeed, in the last two decades a lot has been learned about biomaterials, but, as a matter of fact, the knowledge is still rudimentary. Synthetic vs. natural materials, display higher reproducibility and allow an easy control of mechanical properties, degradation rate, shape, composition, etc. Synthetic materials with different composition have been scrutinized and manipulated using very sophisticated technologies and very complex designs have been conceived in order to mimic the ECM, but results, even if very encouraging, are far from what is requested for an efficient clinical application of tissue engineering. Two major weaknesses can be envisaged in the extensive research activity so far carried out worldwide. The first deals with the simplistic idea that, using a single homogeneous biocompatible material, it could be possible to mimic the multifaceted characteristics of the ECM. Indeed, living organisms are made of diverse materials and cells assembled in order to take advantage of the respective characteristics. Moreover, the concentration and spatial arrangement of every component is not homogeneous throughout the same tissue. Renovated efforts must be directed to emulate the complexity of the extracellular matrix assembling also to favor cell attachment and differentiation. Molecular interactions between cells and scaffold are due to different properties of the material including its chemical composition and concentration, hydrophilicity/hydrophobicity, roughness, porosity, mechanical characteristics and degradation kinetics that should be analogue to those of the native tissue. Microstructure geometry represents an important aspect for selecting material and fabricating optimized scaffolds able to promote the growth of specific cell types [ix]. 3D-microfabricated scaffolds have been manufactured by soft lithography reproducing cardiac ECM structure using different bioartificial materials [ $\left.{ }^{\mathrm{xxviii}}\right]$. Further, cell adhesion can be increased by honeycomb structures with pores slightly larger than cells $\left[{ }^{x \times i x}\right]$. Microstructured scaffolds, 
in fact, are able to increase the elongation efficiency of human mesenchymal cells as well as polycaprolactone (PCL) scaffolds displaying anisotropic geometry obtained by uniaxial stretch $\left[{ }^{x \times x}\right]$. The second weakness is related to the poor knowledge available on the adaptive mechanisms operated by cells after adhesion to scaffolds with specific characteristics. The present study, however, is intended to suggest a novel solution to improve the scaffold technology in order to contribute to vanish, at least in part, the first weakness. To this purpose, a novel class of multitextured scaffolds made of a single inhomogeneously concentrated component, as inspired by the ECM structure made of solid filaments embedded in a hydrated matrix, has been hypothesized. A Wp network of solid filaments has been manufactured by 3D microstereolithography in a biocompatible environment characterized by PEGDa and curcumin. In order to unravel which signals mostly affect cell differentiation, experiments were carried out under low serum condition (FBS 2\%). Results confirmed that the PEGDa-Wp structure emanates a strong array of signals to progenitor cells guiding their 3D spatial multilayered organization and differentiation, as detected by actinin and Cx43 expression. Noteworthily, the ?sarcomeric actinin as well as $\mathrm{C} \times 43$ proteins were widespread throughout the entire cytoplasm and not organized in sarcomeric structures or in gap junctions connecting cells in a head-to-tail manners, respectively. Therefore, a full progenitor cell maturation to cardiomyocyte was not observed. In order to verify whether a more reliable microenvironment could improve hCPC differentiation, cells were cultured for 3 weeks into the 3D-SS. In this context, the hydrogel could play a role in optimizing cell supplying with nutrients and oxygen. In fact, results demonstrated that, in this more complete environment and under standard serum condition (FCS 10\%), cells showed an upregulation of -actinin and a more elongated morphology, although a complete sarcomeric rearrangement in the cytoplasm was not observed. In recent studies, cells grown on hydrogels of fibrin and Matrigel $\left.{ }^{[x \times i}\right]$ or PEG [xxxi] have shown differentiation characteristics of native myocardium. Scaffolds have been credited to provide only mere physical support for stem cell adhesion and proliferation, and geometrical guidance in tissue organization, whereas growth factors [xxiii, xxxiv,$\left.{ }^{x \times x}\right]$, physical stimuli $\left[{ }^{\text {xxxvi }},{ }^{x x x v i i}\right]$ or co-culturing with mature cardiomyocytes [xxiii, xxxviii] have been considered as the major players in driving cell differentiation. Conversely, several studies performed over the years have exhaustively 


\begin{abstract}
Abbreviations
hCPC, Lin- Sca-1+ human cardiac progenitor cells; PGS, poly(glycerolsebacate); PEGDa, polyethylene glycol diacrylate; PEGDa-Wp, PEGDa -Wood pile scaffold; $\mu \mathrm{SL}$ microstereolithography; 3D-SS, 3D- "scaffold into the scaffold"; DAPI , 4',6-diamidino-2phenylindole; WST-[3-(4-lodophenyl)-2-(4-nitrophenyl)-2H-5-tetrazolio]-1,3-benzene disulfonate.
\end{abstract}

\title{
Acknowledgements:
}

The authors are grateful to Dr Elena Romano, Centre for Advanced Microscopy (CAM), Dept. of Biology, University of Rome "Tor Vergata") for the scientific and technical support in the microscopy analysis and Dr Massimiliano Lucci, Department of Physics, University of Rome "Tor Vergata", for the scientific and technical support in the nanoindentation measurements.

\section{Author Disclosure Statement}

No competing financial interests exist. 


\section{References}

[i] Gajarsa JJ, RA Kloner. (2011) Left ventricular remodeling in the post-infarction heart: a review of cellular, molecular mechanisms, and therapeutic modalities. Heart Fail Rev.16:13-21.

[ii] [1] Forrester JS, AJ White, S Matsushita, T Chakravarty, RR Makkar. (2009). New paradigms of myocardial regeneration post-infarction: tissue preservation, cell environment, and pluripotent cell sources. JACC Cardiovasc Interv 2:1-8

[iii] Leor J, Y Amsalem, S Cohen. (2005) Cells, scaffolds, and molecules for myocardial tissue engineering. Pharmacol Ther. 105:151-63.

[iv] Zorlutuna P, N Annabi, G Camci-Unal, M Nikkhah, JM Cha, JW Nichol, A Manbachi, H Bae, S Chen, A Khademhosseini. (2012) Microfabricated biomaterials for engineering 3D tissues. Adv Mater. 24(14):1782-804.

[v] Liu S, P Wang, G Huang, L Wang, J Zhou, TJ Lu, F Xu, et al. (2010) Reaction-induced swelling of ionic gels. Soft Matter 11:449-455.

[vi] Senyo SE, YE Koshman, B Russell. (2007). Stimulus interval, rate and direction differentially regulate phosphorylation for mechanotransduction in neonatal cardiac myocytes. FEBS Lett. 581(22):4241-7

[vii] Marsano A, R Maidhof, LQ Wan, Y Wang, J Gao, N Tandon, G Vunjak-Novakovic. (2010) Scaffold stiffness affects the contractile function of three-dimensional engineered cardiac constructs. Biotechnol. Prog. 26(5):1382-90

[viii] Engler AJ, MA Griffin, S Sen, CG Bönnemann, HL Sweeney, DE Discher.(2004) Myotubes differentiate optimally on substrates with tissue-like stiffness: pathological implications for soft or stiff microenvironments. J Cell Biol.;166(6):877-87.

[ix] Forte G, F Carotenuto, F Pagliari, S Pagliari, P Cossa, R Fiaccavento, A Ahluwalia, G Vozzi, B Vinci, A Serafino, A Rinaldi, E Traversa, L Carosella, M Minieri, P Di Nardo. (2008). Criticality of the biological and physical stimuli array inducing resident cardiac stem cell determination. Stem Cells. 26(8):2093-103.

[x] Zhenqing Li, Jianjun Guan (2011) Hydrogels for Cardiac Tissue Engineering. Polymers, $3(2): 740-761$ 
[xi] Park H, BL Larson, MD Guillemette, SR Jain, C Hua, GC Jr Engelmayr , LE Freed . (2010) The significance of pore microarchitecture in a multi-layered elastomeric scaffold for contractile cardiac muscle constructs. Biomaterials. 32(7):1856-64

[xii] Lutolf PM, PM Gilbert, HM Blau. (2009) Designing materials to direct stem-cell fate. Nature, 462:433-441

[xiii] De Matteis F, F Fanicchia, FR Lamastra, G Stracci, R De Angelis, P Prosposito, F Nanni, M Casalboni. (2014). Nonlinear optical materials by electrospinning technique. J.Appl.Pol. Scie., 131:40913

[xiv] Muskin J, M Ragusa, T Gelsthorpe. (2010). Three-dimensional printing using a photoinitiated polymer. Journal of Chemical Education, 87(5), 512-514.

[xv] Prosposito P, S Melino, M Ciocci, R Francini, F Mochi, F De Matteis, P Di Nardo, S Ksenzov, S Schrader, M Casalboni. (2017). Photolithography of 3D Scaffolds for Artificial Tissue. Materials Science Forum, 879:1519-1523

[xvi] Mochi F, P Prosposito, R Francini, F De Matteis, S Melino, M Ciocci, P Di Nardo, S. Ksenzov, S. Schrader, M. Casalboni. (2016). Advanced biocompatible photolithographic scaffolds for tissue engineering. IET Conference Proceedings 84 (4.)

[xvii] Di Nardo P, Pagliari F. (2017) Cardiac Progenitor Cell Extraction from Human Auricles. Methods Mol Biol., 1553:145-154

[xviii] Anand P, SG Thomas, AB Kunnumakkara, C Sundaram, KB Harikumar, B Sung, ST Tharakan, K Misra, IK Priyadarsini, KN Rajasekharan, BB Aggarwal. (2008). Biological activities of curcumin and its analogues (Congeners) made by man and Mother Nature. Biochem. Pharmacol. 76(11):1590-611

[xix] Harmon ME, D Kuckling, CW Frank. (2003). Photo-cross-linkable PNIPAAm copolymers. 5. Mechanical properties of hydrogel layers. Langmuir 19:10660-10665.

[xx] Drira Z., V. K. Yadavalli. (2013). Nanomechanical measurements of polyethylene glycol hydrogels using atomic force microscopy. J. Mech.Behav.Biomed. Mat. 18:20-28

[xxi] Mouw JK, G Ou, VM Weaver. (2014). Extracellular matrix assembly: a multiscale deconstruction. Nat Rev Mol Cell Biol. 15(12):771-85

[xxii] Traversa E, B Mecheri, C Mandoli, S Soliman, A Rinaldi, S Licoccia, G Forte, F Pagliari, S Pagliari, F Carotenuto, M Minieri, P Di Nardo (2008). Tuning hierarchical 
architecture of 3D polymeric scaffolds for cardiac tissue engineering. Journal of Experimental Nanoscience 3(2):97-110

[xxiii] Soliman S, S Pagliari, A Rinaldi, G Forte, R Fiaccavento, F Pagliari, O Franzese, M Minieri, P Di Nardo, S Licoccia, E Traversa. (2010) Acta Biomater 6:1227-1237.

[xxiv] Mauretti A, A Neri , O Kossover, D Seliktar, P Di Nardo, S Melino.(2016) Design of a Novel Composite H2 S-Releasing Hydrogel for Cardiac Tissue Repair. Macromol Biosci. Jun;16(6):847-58.

[xxv] Wolf MT, CL Dearth, SB Sonnenberg, EG Loboa, SF Badylak. (2015). Naturally derived and synthetic scaffolds for skeletal muscle reconstruction. Adv Drug Deliv Rev. 84:208-21.

[xxvi] Guyette JP, JM Charest, RW Mills, BJ Jank, PT Moser, SE Gilpin, JR Gershlak, T Okamoto, G Gonzalez, DJ Milan, GR Gaudette, HC Ott. (2016). Bioengineering Human Myocardium on Native Extracellular Matrix. Circ Res 118:56-72, [xxvii] Crapo PM, TW Gilbert, SF Badylak. (2011). An overview of tissue and whole organ decellularization processes. Biomaterials 32(12): 3233-3243.

[xxviii] Rosellini E, Vozzi G, Barbani N, Giusti P, Cristallini C. (2010) Three-dimensional microfabricated scaffolds with cardiac extracellular matrix-like architecture. Int J Artif Organs. Dec;33(12):885-94.

[xxix] Arai K, M Tanaka, S Yamamoto, M Shimomura. (2008). Effect of pore size of honeycomb films on the morphology, adhesion and cytoskeletal organization of cardiac myocytes. Colloids Surf A Physicochem Eng Asp 313:530-5

[xxx] Wang ZY, EY Teo, MS Chong, QY Zhang, J Lim, ZY Zhang, MH Hong, ES Thian, JK Chan, SH Teoh. (2013) Biomimetic three-dimensional anisotropic geometries by uniaxial stretch of poly([?] alignment, and myogenic differentiation. Tissue Eng Part C Methods;19: 538-49

[xxxi] Perea-Gil I, C Prat-Vidal, A Bayes-Genis. (2015) In vivo experience with natural scaffolds for myocardial infarction: the times they are a-changin'. Stem Cell Res Ther $6: 248$

[xxxii] Kim DH, Kshitiz, RR Smith, P Kim, EH Ahn, HN Kim, E Marban, KY Suh, A Levchenko. (2012) Nanopatterned cardiac cell patches promote stem cell niche formation and myocardial regeneration. Integr Biol 4:1019-33 
[xxxiii] Forte G, M Minieri, P Cossa, D Antenucci, M Sala, V Gnocchi, R Fiaccavento, F Carotenuto, P De Vito, PM Baldini, M Prat, P Di Nardo. (2006). Hepatocyte growth factor effects on mesenchymal stem cells: proliferation, migration, and differentiation. Stem Cells 24:23-33

[xxxiv] Xaymardan M, L Tang, L Zagreda, B Pallante, J Zheng, JL Chazen, A Chin, I Duignan, P Nahirney, S Rafii, T Mikawa, JM Edelberg. (2004) Platelet derived growth factor-AB promotes the generation of adult bone marrow derived cardiac myocytes. Circ Res 94:E39-45

[xxxv] Behfar A, S Yamada, R Crespo-Diaz, JJ Nesbitt, LA Rowe, C Perez-Terzic. (2010).Guided cardiopoiesis enhances therapeutic benefit of bone marrow human mesenchymal stem cells in chronic myocardial infarction. J Am Coll Cardiol 56:721-34. [xxxvi] Guan J, F Wang, Z Li, J Chen, X Guo, J Liao, NI Moldovan . (2011). The stimulation of the cardiac differentiation of mesenchymal stem cells in tissue constructs that mimic myocardium structure and biomechanics. Biomaterials 32:5568e80.

[xxxvii] Mooney E, JN Mackle, DJ Blond, E O'Cearbhaill, G Shaw, WJ Blau, FP Barry, V Barron, JM Murphy. (2012) The electrical stimulation of carbon nanotubes to provide a cardiomimetic cue to MSCs. Biomaterials 33:6132e9

[xxxviii] Ramkisoensing AA, DA Pijnappels, J Swildens, MJ Goumans, WE Fibbe, MJ Schalij, AA de Vries, DE Atsma. (2012) Gap junctional coupling with cardiomyocytes is necessary but not sufficient for cardiomyogenic differentiation of cocultured human mesenchymal stem cells. Stem Cells 30(6):1236-45.

[xxxix] Leigh SJ, HT Gilbert, IA Barker, JM Becker, SM Richardson, JA Hoyland, JA Covington, AP Dove. (2012) Fabrication of 3-dimensional cellular constructs via microstereolithography using a simple, three-component, poly(ethylene glycol) acrylate-based system. Biomacromolecules 14:186-192.

[xl] Cristallini C, E Cibrario Rocchietti, L Accomasso, A Folino, C Gallina, L Muratori, P Pagliaro, R Rastaldo, S Raimondo, S Saviozzi, AE Sprio, M Gagliardi, N Barbani, C Giachino. (2014). The effect of bioartificial constructs that mimic myocardial structure and biomechanical properties on stem cell commitment towards cardiac lineage. Biomaterials 35:92-104. 
[xli] Zhang YS, A Arneri, S Bersini, SR Shin, K Zhu, Z Goli-Malekabadi, J Aleman, C Colosi, F Busignani, V Dell'Erba, C Bishop, T Shupe, D Demarchi, M Moretti, M Rasponi, MR Dokmeci, A Atala, A Khademhosseini. (2016) Bioprinting 3D microfibrous scaffolds for engineering endothelialized myocardium and heart-on-a-chip. Biomaterials 110:45-59 
Figure legends
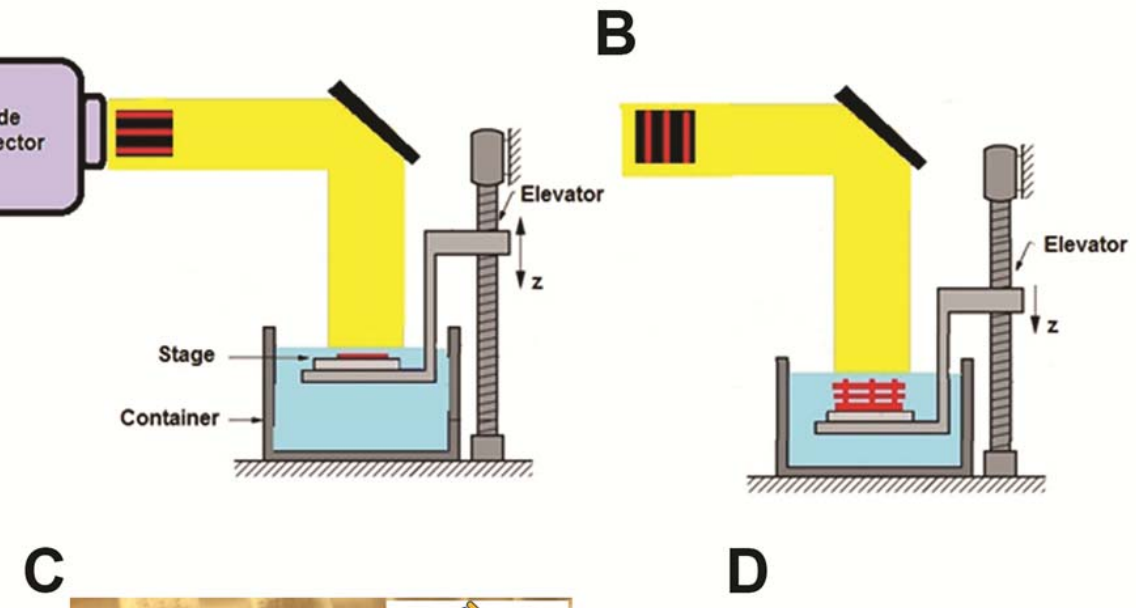

C

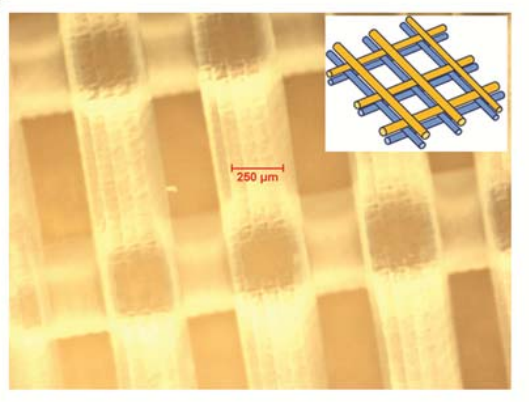

D

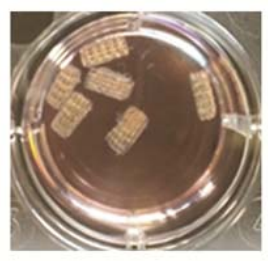

Fig. 1. Experimental set-up for Microstereolithography (?SL): (A) at the start of the process, in which the initial layer is added to the stage; (B) after several layers have been added so that the structure gradually takes form. Curcumin is used as absorbing dye. (C) Light micrograph of a PEGDa template manufactured by lsL process. The sketch of a Woodpile (WP)-structure composed by two monolayers is shown in the inset. (D) Micrograph of the 3D PEGDa-Wp structures in a 24 multiwell plate. 


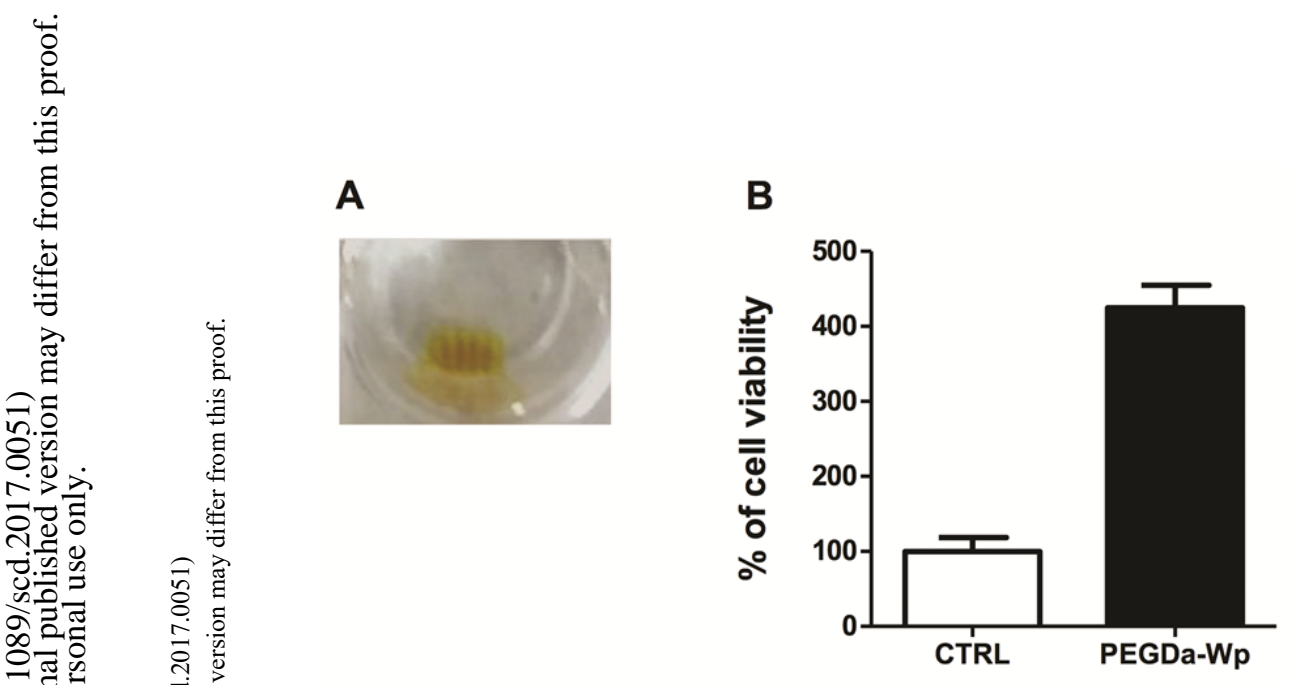

Fig. 2. hCPCs adhesion and proliferation on PEGDa-Wp structures. (A) hCPCs (Lin- Sca-1+ human cardiac precursor cells) grown for 4 days on a PEGDa-Wp structure after Wst-1 cell viability assay; (B) cell proliferation of hCPCs at time 0 (CTRL) and after 4 days; (C) number of hCPCs that adhered to the PEGDa-Wp structure at day 4 of growth. Each bar represents the mean $\pm S D$ of five experiments. 


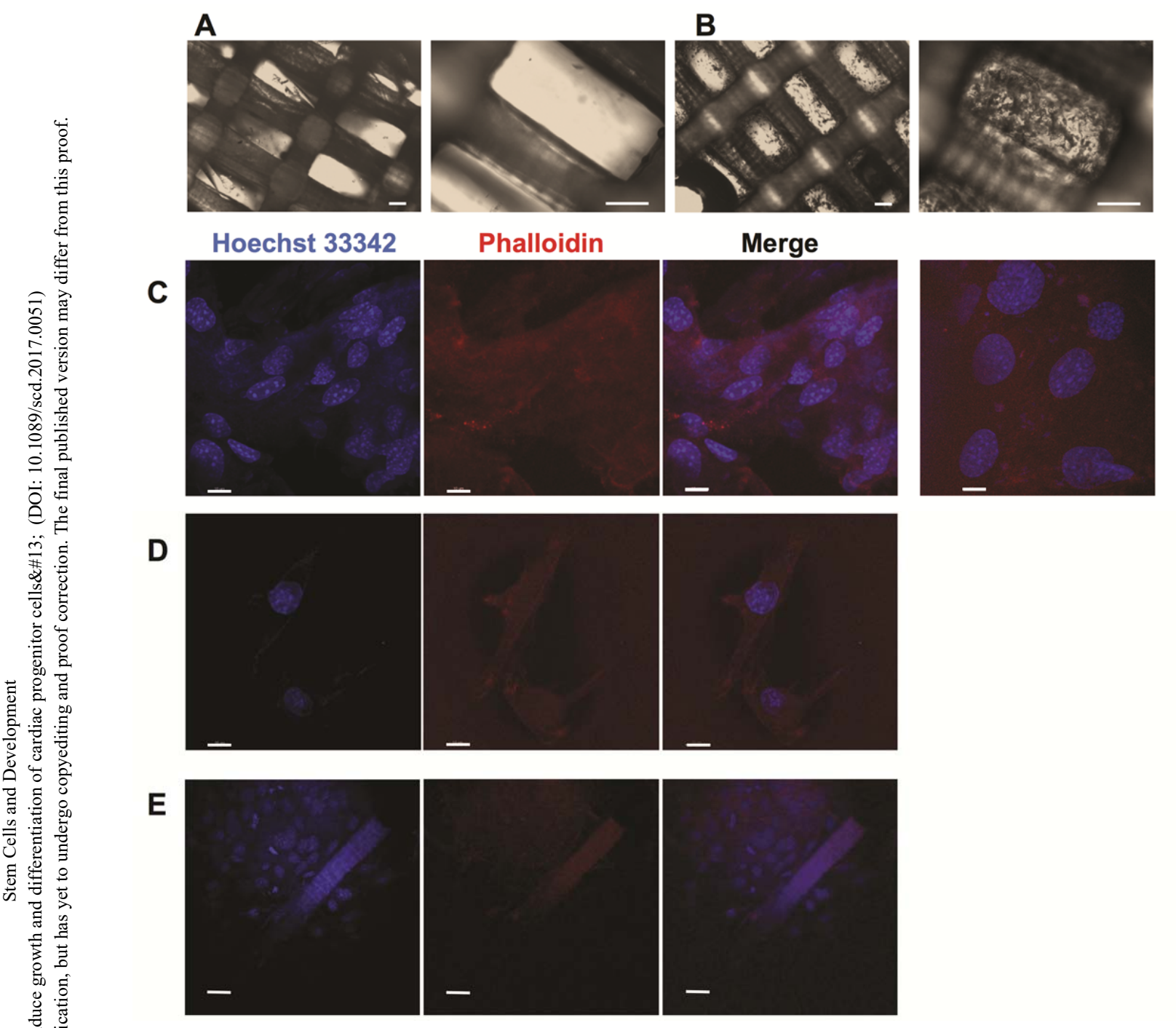

Fig. 3. hCPCs adhesion, alignment and elongation are improved by PEGDa-Wp structures. Light micrograph of PEGDa-WP structures in (A) the absence and (B) the presence of hCPCS after 8 days culture; (C) and (D): Confocal micrographs of hCPCs cultured for 8 days on PEGDa-Wp structures. Nuclei (blue) are stained with Hoechst 33342, while the cytoskeleton (red) is stained with Alexa fluor-Phalloidin. Scale bars: $10 \mu \mathrm{m}$ in (C) and (D) and $30 \mu \mathrm{m}$ in (E). 


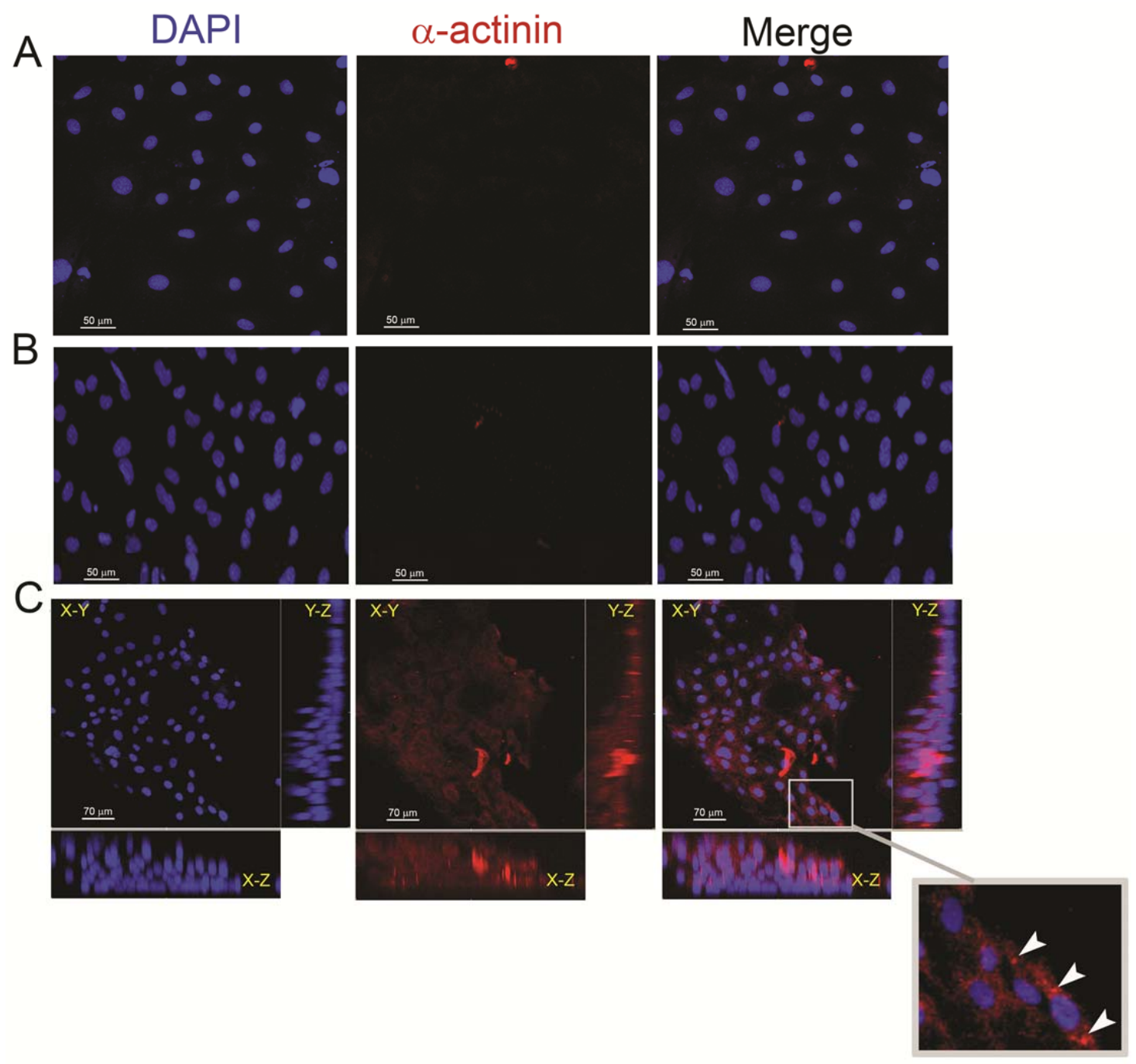

Fig. 4. hCPCs cardiac commitment on PEGDa-WP structures. Confocal micrographs of hCPCs cultured for 12 days (A) in chamber slides; (B) on 2D PEGDa-Wp and (C) on 3D PEGDa-Wp structures. (A) and (B): No ?-actinin expression (red) is apparently present in cell cytoplasm. Nuclei (blue) are stained by DAPI. (C) Confocal X-Y, X-Z, Y-Z sections in max intensity projection mode (MIP modality). ??-actinin (red) is expressed in multilayered hCPCs cultured on 3D PEGDa-Wp structures. A magnification $(10 \times 10)$ of the image $(\mathbf{B})$ is shown in the panel right-bottom. Arrowheads indicate a characteristic cytosol ?-actinin accumulation suggesting protein processing by Golgi complexes. 
Page 27 of 32

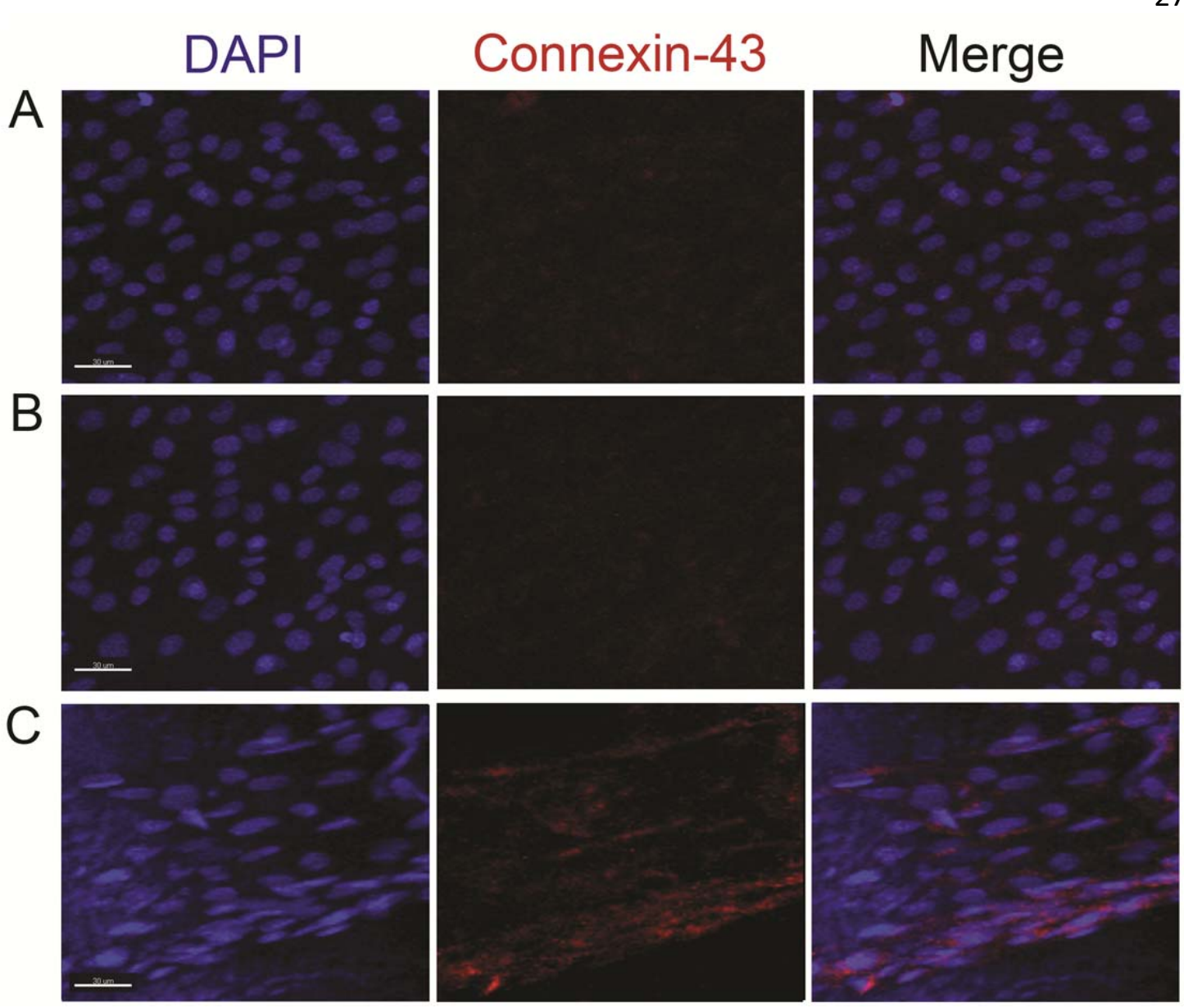

Fig.5. PEGDa-WP structures enhance connexin 43 expression in hCPCs. Confocal micrographs of connexin 43 expression in hCPCs cultured for 3 weeks in chamber slides in the absence $(A)$ or in the presence of 2D PEGDa-Wp (B) or 3D PEGDa-Wp (C) structures. A widespread connexin-43 specific signal (red) is detectable throughout the entire cytoplasm of hCPCs cultured on 3D PEGDa-Wp structures, only (C). Nuclei (blue) are stained with DAPI. Scale bars: 30 ? $m$ in A, B and C 

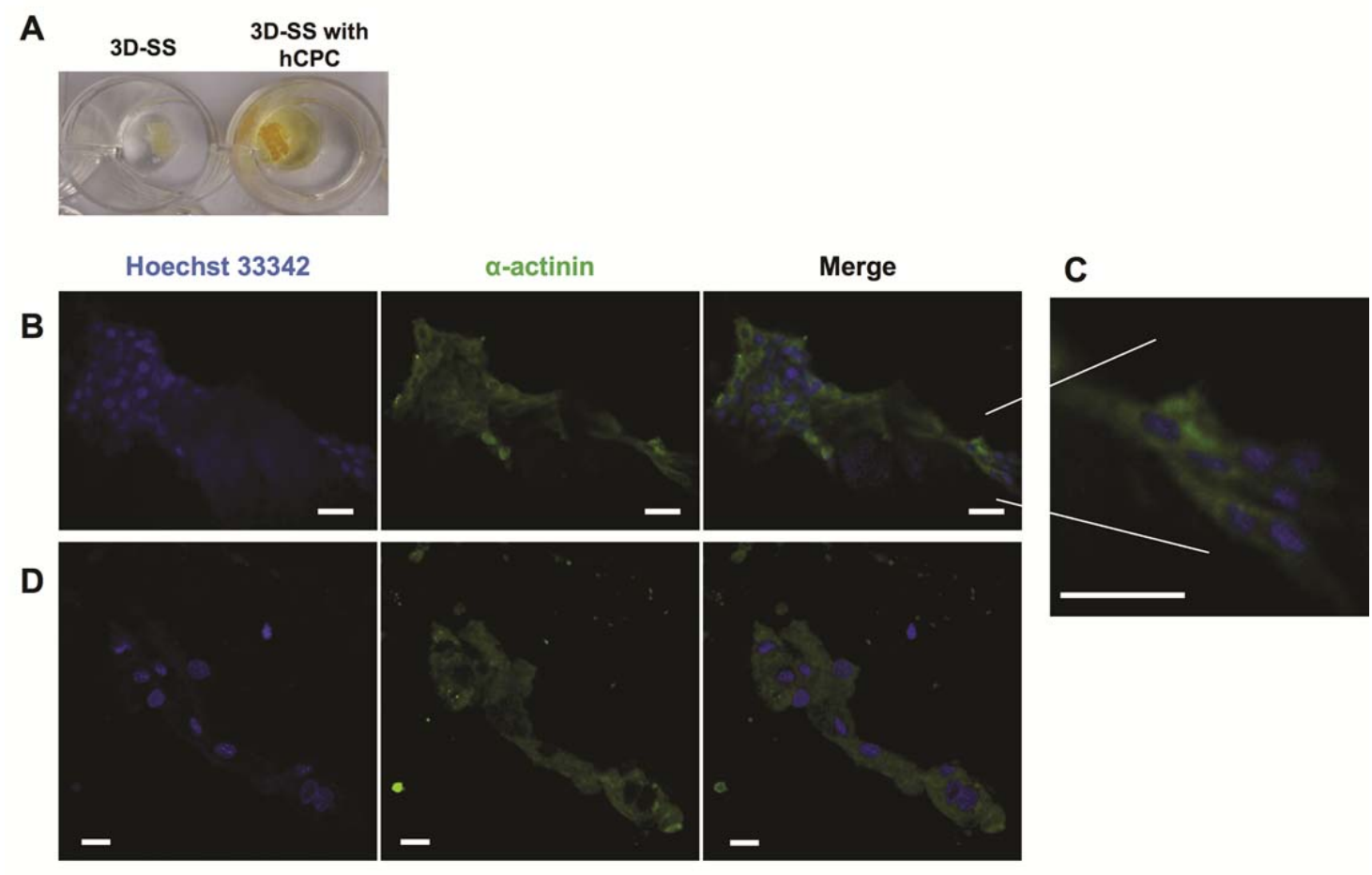

Fig. 6. 3D-SS composite scaffold showing a differentiation-inducing potential on hCPCs. (A) 3D-SS with and without hCPCs cultured for 3 weeks after Wst-1 cell viability assay; (B) and (D) confocal micrographs of hCPCs grown on 3D-SS after 3 weeks culture. Nuclei (blue) are stained with Hoechst 33342, while the $\alpha$-actinin expression is shown in green. (C) magnification (3.5×3.5) of a selected region of (B). Scale bars: $20 \mu \mathrm{m}$. 


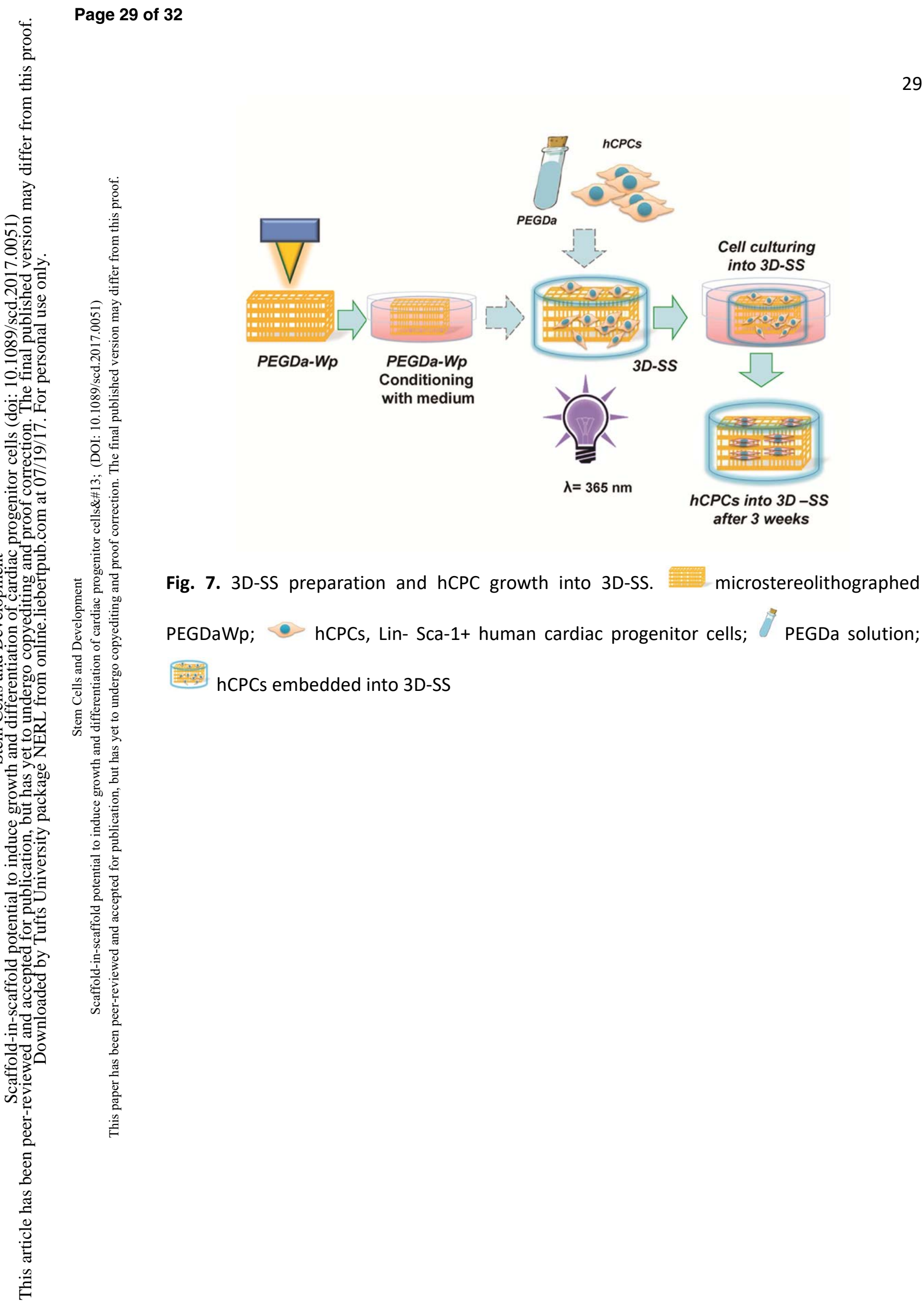


Supplementary Materials

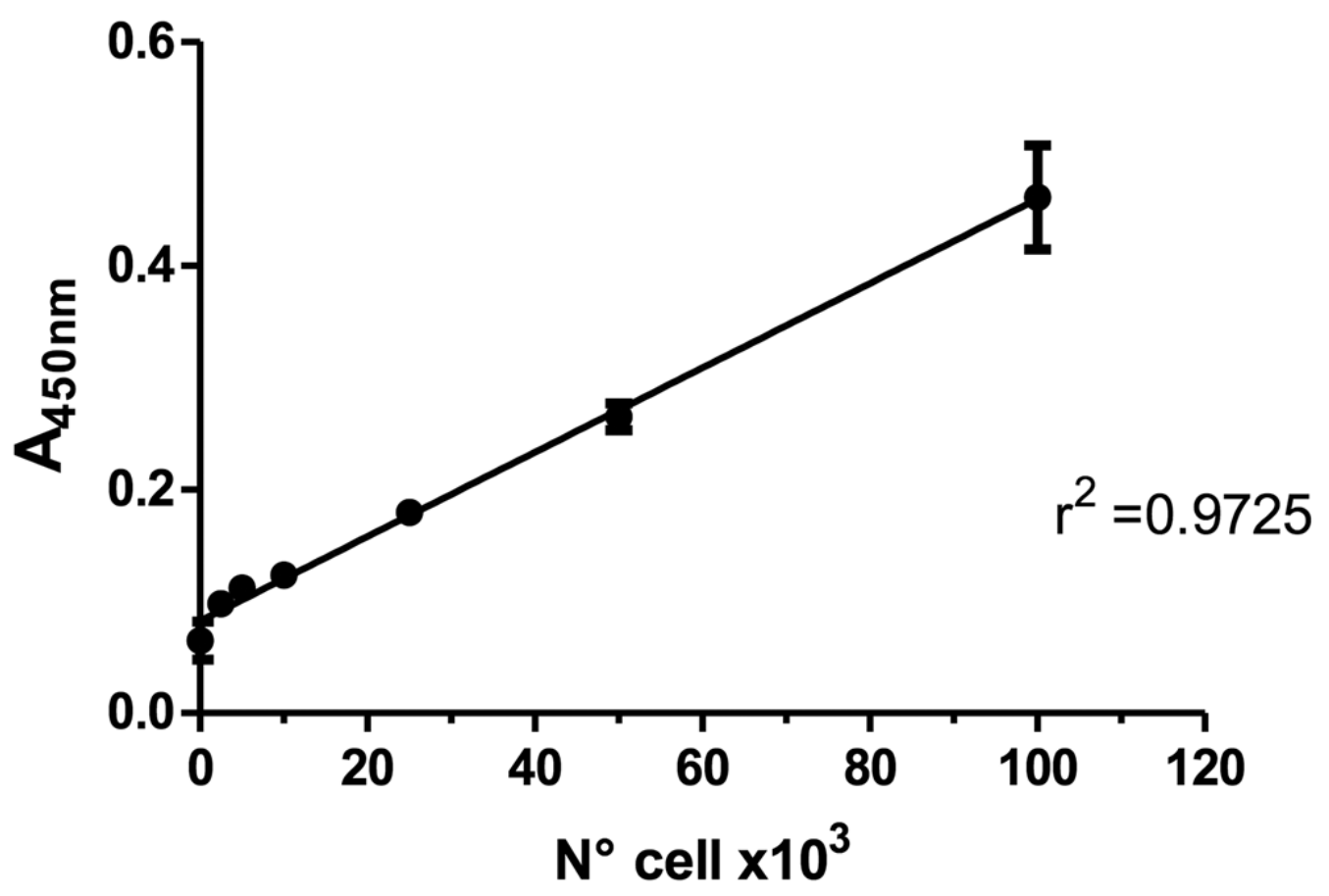

Fig. 1S. Calibration curve for cell counting analysis. Correlation curve between the number of cells and the absorbance measured at $450 \mathrm{~nm}$ obtained by the colorimetric cell viability Wst-1 assay. 


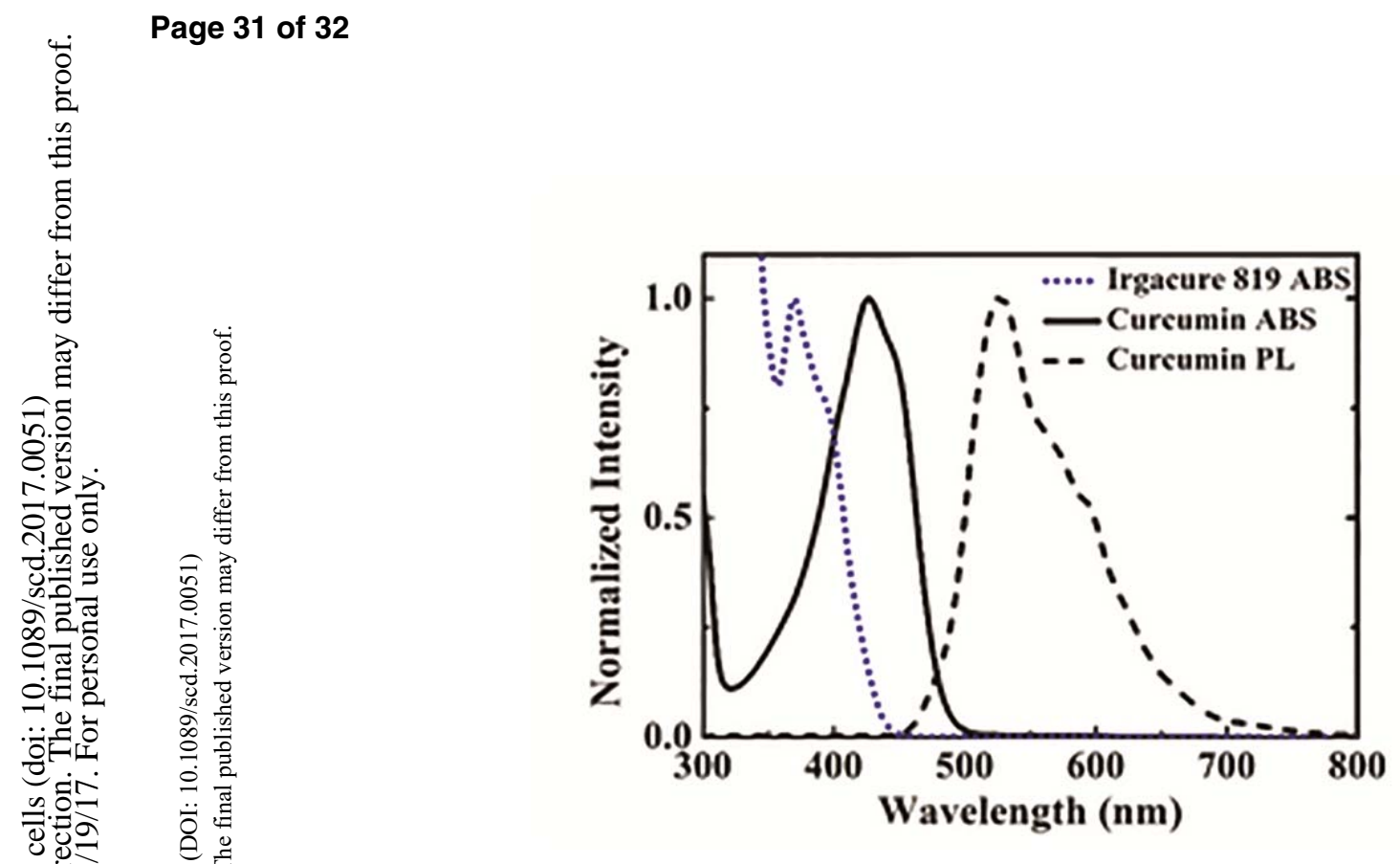

Fig. 2S. Curcumin and Irgacure 819 optical properties. 

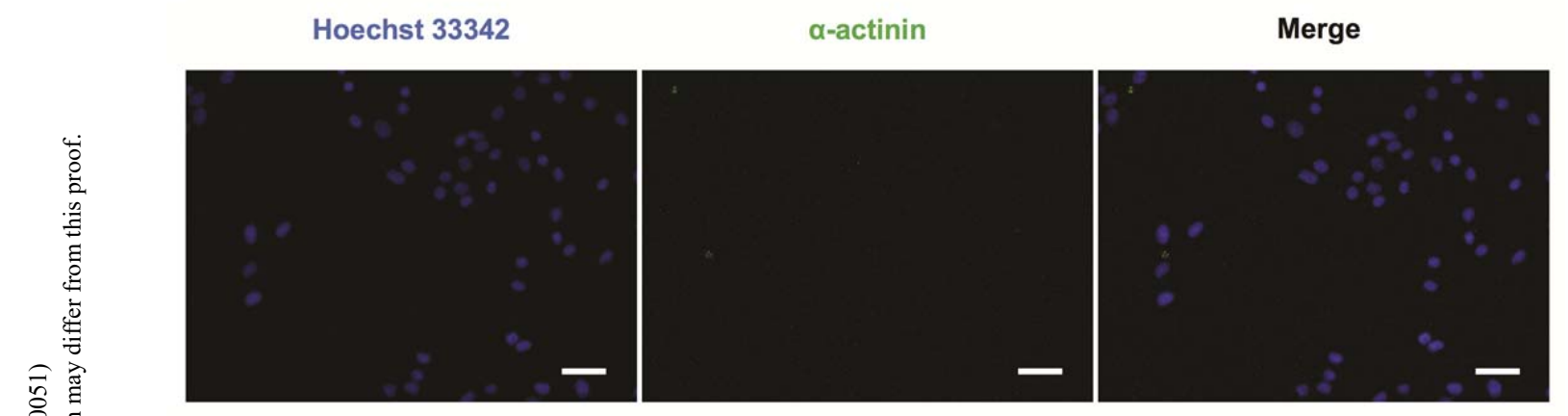

Merge

Fig. 3S. Immunofluorescence micrographs of hCPCs cultured on plate. hCPC nuclei are stained by Hoechst 33342 (blue) and the ?-actinin protein is stained in green. After four days of growth on plate the ?-actinin protein expression is not detectable. Scale bars: 50 $\mu \mathrm{m}$. 